\title{
The effects of HIV self-testing on the uptake of HIV testing and linkage to antiretroviral treatment among adults in Africa: a systematic review protocol
}

\author{
Bernard Njau ${ }^{1 *}$, Damian J. Damian', Leila Abdullahi ${ }^{2,3}$, Andrew Boulle ${ }^{1}$ and Catherine Mathews ${ }^{1,4}$
}

\begin{abstract}
Background: HIV is still a global public health problem. More than $75 \%$ of HIV-infected people are in Africa, and most of them are unaware of their HIV status, which is a barrier to accessing antiretroviral treatment. Our review aims, firstly, to determine whether HIV self-testing is an effective method to increase the uptake of testing, the yield of new HIV-positive diagnoses, and the linkage to antiretroviral treatment. Secondly, we aim to review the factors that facilitate or impede the uptake of HIV self-testing.

Methods/design: Participants will be adults living in Africa. For the first aim, the intervention will be HIV self-testing either alone or in addition to HIV testing standard of care. The comparison will be HIV testing standard of care. The primary outcomes will be (i) uptake of HIV testing and (ii) yield of new HIV-positive diagnoses. The secondary outcomes will be (a) linkage to antiretroviral (ARV) treatment and (b) incidence of social harms. For the second aim, we will review barriers and facilitators to the uptake of self-testing. We will search PubMed, Cochrane Central Register of Controlled Trials, Scopus, Web of Science, WHOLIS, Africa Wide, and CINAHL for eligible studies from 1998, with no language limits. We will check reference lists of included studies for other eligible reports. Eligible studies will include experimental and observational studies. Two authors will independently screen the search output, select studies, and extract data, resolving discrepancies by consensus and discussion. Two authors will use Cochrane risk of bias tools for experimental studies, the Newcastle-Ottawa Quality Assessment Scale for observational studies, and the Critical Appraisal Skills Programme (CASP) quality assessment tool for qualitative studies.
\end{abstract}

Discussion: Innovative and cost-effective community-based HIV testing strategies, such as self-testing, will contribute to universal coverage of HIV testing in Africa. The findings from this systematic review will guide development of selftesting policy in African countries.

Systematic review registration: PROSPERO CRD42015023935

Keywords: HIV self-testing, Uptake, Yield, Barriers, Facilitators, Social harms, Africa

\footnotetext{
* Correspondence: biesein2007@gmail.com

${ }^{1}$ School of Public Heath and Family Medicine, University of Cape Town, Cape

Town 7925, South Africa

Full list of author information is available at the end of the article
} 


\section{Background}

Globally, an estimated 35.0 million people are living with HIV, with more than 2.1 million (1.9 million-2.4 million) new HIV infections and 1.5 million deaths in 2013 [1]. Africa remains the most affected region, contributing more than two thirds of the global burden of HIV [2]. The UNAIDs/WHO has set a "90-90-90" global target to be reached by 2020 . The global target stipulates that $90 \%$ of adults will know their HIV status, $90 \%$ of HIV positive will receive sustained antiretroviral (ARV) treatment, and $90 \%$ of those who are on ARV treatment will achieve viral load suppression by 2020 [3].

Despite substantial efforts to increase HIV testing, particularly in populations with generalized HIV epidemics, testing coverage in Africa is low. A recent review of Demographic and Health Surveys reported that across 29 Sub-Saharan Africa (SSA) countries, only $15 \%$ of adults received HIV test result in the past 12 months [4]. The observed low coverage is a critical barrier to scaling up HIV prevention, care, and treatment interventions. In addition, evidence suggests that most HIV-positive people in Africa seek late entry to ARV treatment and the delay has adverse impact on treatment outcomes, including high mortality, avoidable morbidity, and transmission of the virus $[5,6]$.

Evidence shows that HIV self-testing (HIVST) has the potential to empower non-testers to know their HIV status by overcoming facility-based barriers to HIV testing [7]. However, HIV policy makers have reservations about the introduction of self-testing and raise concerns regarding HIVST. The concerns vary from lack of policies and regulatory systems, quality of HIVST kits, ethical and human rights issues, and knowledge gaps pertaining to HIVST [8-10].

Apart from general concerns described above, there are several barriers associated with uptake of HIVST. Perceived accuracy of self-testing kits is a major barrier to uptake of HIVST, with evidence of uncertainty on perceptions of the accuracy of self-test kits [11]. The invasive nature of a finger prick for obtaining a blood sample is another barrier, particularly for blood-based self-testing because some people fear needle pricks [12]. The cost of buying self-test kits is another barrier. Most consumers have to pay for the self-test kits, ranging from US\$ 4.8 to US\$ 40, which may not be affordable to most people in low- and middle-income countries (LMICs), including SSA [8, 13]. Finally, illiteracy is another barrier associated with uptake of HIVST. Evidence shows that illiterate participants are less likely to undertake self-testing compared to their literate counterparts, particularly in an unsupervised self-testing strategy [13]. The current evidence on barriers to uptake of HIVST is from studies conducted in high-income settings and only four studies from Africa.
Three systematic reviews provided evidence on selftesting, globally. The reviews focused on HIVST strategies in low- and high-risk populations and acceptability of HIVST in high- and low-income countries. Pai et al.'s review reported a high acceptability, ranging from 74 to $96 \%$ in high- and low-income countries. In addition, the review observed a higher preference for self-testing compared to facility-based testing and oral-based rather than blood-based self-testing [14]. Krause et al.'s review found that 50 to $60 \%$ of participants were first-time testers. In addition, the review provided evidence of high performance accuracy rates of $99 \%$ among laypersons compared to health-care providers. The review concluded that HIVST has a potential to reach first-time testers with high performance accuracy rates among laypersons [15]. Suthar et al.'s review, which compared different community-based HIV testing approaches, reported participant's high uptake of self-testing of $86.9 \%$ compared with $62 \%$ of a school-based HIV testing. The review concluded that community-based HIV approaches, including self-testing, have high rate of testing uptake [16]. The current reviews focused mainly on studies from high-income settings, with only four studies (Malawi $n=3$; Kenya $=1$ ), were from African countries. The four studies lack evidence on yield of new HIV-positive diagnoses, post-test linkage to ARV treatment, and incidence of social harms post-testing [17]. However, since the last reviews in 2014, five new African studies on self-testing have been published.

Of importance is the fact that apart from WHO recommendation for introduction of HIVST as a strategy to increase universal coverage of HIV testing and counseling (HTC) [18], most African countries lag behind in terms of integration of HIVST in their national HTC guidelines [9]. In addition, there is a concern that with HIV self-testing, linkage to treatment (i.e., HIV-positive clients receiving sustainable ARV treatment) might be compromised [19]. A key challenge with HIVST implementation is ensuring people who test positive are not alienated from health services where ARV treatment is provided, because of the privacy and lack of post-test counseling associated with self-testing [8]. In order to answer this pertinent question, we propose to conduct this review. The aim of this study is to review the existing evidence on the effects of HIVST on the uptake of testing, the yield of new HIV-positive diagnoses, linkage to ARV treatment, incidence of social harms, and the factors that facilitate and impede the uptake of HIVST among adults in Africa.

\section{Aims}

The first aim is to evaluate the effectiveness of HIV selftesting interventions on (i) the uptake of HIV testing, (ii) 
the yield of new HIV-positive diagnoses, linkage to ARV treatment, and (iii) incidence of social harms among adults in Africa.

The second aim is to review the barriers and facilitators to the uptake of self-testing among adults in Africa.

\section{Methods}

This review has been registered in the PROSPERO International Prospective Register of systematic reviews (http://www.crd.york.ac.uk/PROSPERO), registration number CRD42015023935 [20].

\section{Eligibility criteria}

\section{Study designs}

For the review of the intervention effects, experimental studies including randomized controlled trials (RCTs), controlled before/after studies, and interrupted time series studies will be considered for inclusion in this review. Experimental studies will assess the effects on uptake, yield, incidence of social harms, and linkage to ARV treatment. In addition, given that this is an emerging area of research, we will conduct secondary supplementary analysis of observational studies that assessed the outcomes of interest in this review. These studies will be included in a separate section of the review results.

For the review of barriers and facilitators, we will include predominantly observational studies which include data on the factors which facilitate or impede the uptake of selftesting. We will employ a broad definition of observational studies and include all studies that utilize qualitative methods for data collection (i.e., focus groups, in-depth interviews, observation, and review of documents) and analysis by the contextual attributes, such as factors associated with gender, culture, ethnicity, and geographical settings.

\section{Participants}

The study participants are adults (males and females) from African countries.

\section{Intervention}

The intervention of this study is HIV self-testing. HIV self-testing refers to a process whereby an individual, who is willing to know his/her HIV status, collects his/ her own specimen, performs the HIV test, and interprets the results in private at home or in other settings.

For the review of the intervention effects, the intervention condition is HIV self-testing, either alone or in addition to the standard of care in HIV testing. The comparison condition is HIV testing standard of care. This might involve (1) provider-administered testing, (2) doorto-door testing, (3) mobile testing, (4) index testing, (5) work place testing, and (6) client-initiated testing or some combination of these approaches.

\section{Outcomes}

For the review of the intervention effects, the primary outcomes are uptake of testing and yield of new HIV-positive diagnoses among adults in African countries. The primary outcomes are defined as:

- Uptake: the proportion of individuals who underwent HIV testing and received their test results over those who were offered HIV testing [21].

- Yield of new HIV-positive diagnoses: the proportion of individuals who were newly diagnosed with HIV positive over those who were offered HIV testing [22, 23].

The secondary outcomes are defined as:

- Linkage into ARV treatment: proportion of newly diagnosed HIV-positive adults who are enrolled in ARV treatment at any point in time post-testing over all newly diagnosed HIV-positive adult participants [24].

Social harm: proportion of participants who report any episode of harm during or after HIV testing (e.g., intimate partner violence, coercive testing by a partner, or suicide) [25].

Editorials, reviews, perspectives, and studies not evaluating self-testing strategies (e.g., home-based testing) will be excluded. Studies, which will not clearly define the type of HIV testing strategies or include subjects below 18 years old will also be excluded. We will not consider studies that included health-care providers. Any disagreements in study inclusion/exclusion will be resolved at a meeting between reviewers. For the qualitative studies, we will collect additional information concerning (a) barriers to the uptake of self-testing, (b) facilitators to the uptake of self-testing, and (c) experiences of adults who had participated in self-testing in any African countries.

\section{Setting}

We will include studies conducted in any country on the African continent.

\section{Information sources}

\section{Electronic databases}

We will conduct two separate search strategies for aims one and two. For the review of the intervention effects, a comprehensive search strategy will be developed to identify both published and unpublished articles with no language restrictions from 1998 to 31st December 2015. 
This search restriction is used because since 1998, we have seen the emergence of advance developments of rapid HIV diagnostic tests (RDTs) including self-testing [26]. The review will search for related studies in PubMed, the Cochrane Central Register of Controlled Trails (CENTRAL), the Cochrane Database of Systematic Reviews (CDSR), Databases of Abstracts of Reviews of Effectiveness (DARE), Social Sciences Citation Index, Web of Science, and African Index Medicus. In addition, we will search websites and databases for gray materials such as World Health Organization Library Information System (WHOLIS), WHO Global Index Medicus, the Joint United Nations Programme on HIV/ AIDS (UNAIDS resource library), Alliance of Health Policy and Systems Research, and The World Bank. The search strategies for electronic databases will incorporate Medical Subject Headings (MeSH), free-text terms, and comprehensive African search filter that will be adapted to suit each individual database using applicable controlled vocabulary $[27,28]$. We will also check reference lists of included studies for other eligible reports.

For the review of barriers and facilitators, we will search for related studies in CINAHL and MEDLINE electronic databases using guidelines developed by the Cochrane Qualitative Research Methods Groups for searching for qualitative evidence [29]. Previous qualitative work has demonstrated that CINAHL and MEDLINE are the most important resource for qualitative evidence [30]. In addition to the abovementioned databases, we will search other resources for related articles, contact experts in the field, gray literature, and scan reference lists of relevant studies.

\section{Search strategy}

For the review of the intervention effects, we will use various $\mathrm{MeSH}$ and search terms such as "adult," "HIV," "Human Immunodeficiency Virus," "AIDS," "Acquired Immunodeficiency Syndrome," "self testing," "HIV selftesting," "HIVST," "testing," "counseling," "provider-administered testing," "uptake," "yield," "prevalence," "HIV positivity," "linkage," "care," "treatment," Africa," and "Africa South of the Sahara". Searches will combine with the names of each country in Eastern, Northern, and Southern Africa by using the Boolean operators "OR" or "AND" (Additional file 1).

For the review of barriers and facilitators, we will use search terms for Boolean search strategy such as "adult," "HIV," "Human Immunodeficiency Virus," "AIDS," "Acquired Immunodeficiency Syndrome," "self testing," "HIV self-testing," "HIVST," "barriers," "facilitators," "HIV self-testing experiences," Africa," and "Africa South of the Sahara". We will use various combinations of these terms with the search engines.

\section{Study records}

\section{Data management}

All search results will be merged into reference management software EndNote, and duplicate records of the same report will be removed.

\section{Selection process}

Full copies of articles identified by the search, and which meet the inclusion criteria, based on the title and abstract will be obtained for data synthesis. Firstly, two reviewers will independently apply the inclusion criteria to the results of the searches, based on the titles and abstracts alone.

\section{Data collection process}

Two reviewers will extract data using a pre-designed data extraction forms and summarize the most important information from each study independently. A third reviewer will be consulted to resolve any differences of opinion between the two reviewers if they may arise. We will conduct a pilot trial of both data extraction forms to check its adequacy and make changes if necessary.

\section{Data items}

Where possible, extracted data will include study details, setting of the study (e.g., city/country/or rural/urban or facility-based/community-based), year of publication-1998 to date, and type of HIV self-testing (e.g., supervised or unsupervised) (Additional files 2 and 3).

\section{Risk of bias in included studies}

Two reviewers will code each included study using Cochrane risk of bias tools for RCT studies [31] and the Newcastle-Ottawa Quality Assessment Scale for observational studies [32]. This will be supplemented with the Effective Practice and Organisation of Care (EPOC) "Risk of bias" guidance to assess the risk of bias of nonrandomized studies [33]. Studies will be assessed on sequence generation; allocation concealment; blinding of participants, personnel, and outcome assessors; incomplete outcome data; selective outcome reporting; and other sources of bias and rated as low risk/or adequate, high risk/ or inadequate, and unclear [34] (Additional file 2).

We will use the Critical Appraisal Skills Programme (CASP) quality assessment tool to assess the methodological quality of the qualitative studies $[35,36]$. This tool includes the following 14 questions: (1) Is this study qualitative research? (2) Are the research questions clearly stated? (3) Have ethical issues been taken into consideration? (4) Is the qualitative approach clearly justified? (5) Is the approach appropriate for the research question? (6) Is the study context clearly described? (7) Is the role of the researcher clearly described? (8) Is the sampling method clearly described? (9) Is the sampling 
strategy appropriate for the research question? (10) Is the method for data collection clearly described? (11) Is the data collection method appropriate for the research question? (12) Is the method of analysis clearly described? (13) Is the chosen analytical approach suitable for addressing the research question? and (14) Are the claims made supported by sufficient evidence? [36]. We will conduct a pilot trial on four included studies to assess the feasibility of the use of the tool and ensure integrity of the assessment. The quality assessment for risk of bias will be cross-checked by a third reviewer for discrepancies.

\section{Quantitative data analysis and synthesis}

We will express the results of each study as risk ratios with corresponding $95 \%$ confidence intervals. We will combine the estimates according to the study design; that is, we will pool estimates for each stratum (by design of the study). We will not combine data across different types of design. Random effects meta-analysis will be preferred due to anticipated heterogeneity in study results. The study will use the log relative risks for intervention studies using the generic inverse variance method in Cochrane Review Manager [37]. If we encounter variation in reported outcome measures between studies, median effect sizes will be presented (with a range).

\section{Dealing with missing data}

The study will make efforts to contact corresponding authors to request for clarification of all relevant information in case of missing data. In case the corresponding author fails to respondent within a week of requesting for information, other author(s) will be contacted (copying the first author). A full description of missing data and drop-outs for each included study will be elaborated in the risk of bias table and discuss the extent to which the missing data could alter the results. The study will conduct sensitivity analyses to assess the effect of missing data on the primary meta-analyses. Ongoing projects will be classified as studies awaiting classification.

\section{Unit of analysis issues}

In case of investigators report on cluster-randomized trial data as if randomization was performed at individual level rather than the cluster, a request to study authors for the intra-cluster correlation coefficient (ICC) will be submitted. In case of failing to obtain the information, the study will seek external ICC estimates from similar studies or available resources [38]. The study will use the established ICC to reanalyze the trial data to obtain approximate correct analyses according to description in the Cochrane Handbook for Systematic Reviews of Interventions [34]. A sensitivity analyses to assess the potential bias that may have occurred as a result of the inadequately controlled clustered trials will be performed. Further, the study will perform sensitivity analyses if the ICCs were obtained from external sources to assess the potential biasing effects of inadequately controlled cluster-randomized trials [39].

\section{Assessment of heterogeneity}

For the review of the intervention effects, heterogeneity will be assessed by inspecting a forest plot initially and later through the Cochran's chi-square test using a $10 \%$ level of significance cut-off, and $I^{2}$ statistic where values of 25,50 , and $75 \%$ reflect low, medium, and high heterogeneity, respectively [34, 37]. For the review of barriers and facilitators, we will record differences across the studies with regard to settings, participants, barriers, and facilitators to the uptake of self-testing, for example, and these will inform the analyses.

\section{Sensitivity analysis}

In case the identified studies are similar enough that it would be sensible to combine them in a meta-analysis, we will conduct sensitivity analyses to investigate the robustness of the results to risk of bias (i.e., omitting any studies with high risk of bias) and method of metaanalysis (i.e., random effects vs. fixed effect). We will conduct sensitivity analysis to (i) evaluate the effect of excluding studies unable to meet each quality criterion affect the overall estimate and (ii) evaluate the change in the results if only high-quality studies where included.

\section{Assessment of reporting biases}

The study will employ strategies to search for and include relevant unpublished studies in order to reduce publication bias. These strategies will include searching the gray literature, including conference proceedings (e.g., 1st International symposium on self-testing for HIV and International AIDS Society) and prospective trial registration database to overcome time-lag bias. A funnel plot will be used to investigate the risk of publication bias by intervention type, provided 10 or more studies are included in the analysis for each intervention type. The funnel plot will be critically examined for asymmetry by both visually and use of formal tests.

\section{Grading the quality of evidence}

The study will use the Grading of Recommendations Assessment, Development and Evaluation (GRADE) approach to assess the quality of evidence related to each of the primary and secondary outcomes for the review of the intervention effects. The GRADE approach results in an assessment of the quality of a body of evidence will be categorized as high, moderate, low, and very low [40]. High-quality evidence refers to "further research is very 
unlikely to change our confidence in the estimate of effect." Moderate-quality evidence implies that "further research is likely to have an important impact on our confidence in the estimate of effect and may change the estimate." Low-quality evidence means "further research is very likely to have an important impact on our confidence in the estimate of effect and is likely to change the effect." Evidence is considered of very low quality if "we have very little confidence in the effect estimate." Two authors (BN and DD) will independently assess the quality of evidence as implemented and described in the GRADE profiler (GRADEpro) software [41].

For the review of barriers and facilitators, we will assess the certainty of findings from qualitative studies using the CerQual (certainty of qualitative evidence) approach. Certainty refers to how likely it is that the review finding happens in a context of the included studies and could happen elsewhere. In this approach, assessment of certainty is based on two factors: the methodological quality of individual studies and the plausibility of each study finding. We will use an adaptation of the CASP tool for qualitative studies to assess the methodological quality of individual studies [36]. We will also assess the plausibility of each study finding by looking at the extent to which we are able to identify a clear pattern across the individual study data. This pattern could include, for example circumstances where findings are consistent across multiple contexts or where the review finding incorporates explanations for any variability across individual studies. As a final step, we will prepare summary of findings of the qualitative evidence synthesis. The summary tables will provide key findings, the certainty of evidence for each finding, and explanation of the assessment of the certainty of the qualitative evidence [36]. We will use three levels to indicate the certainty of the qualitative evidence-high, moderate, and low. High certainty will refer to findings drawn from generally well-conducted studies with high levels of plausibility. Moderate certainty will refer to findings drawn from studies with concerns related to either plausibility or methodological quality. Low certainty will refer to findings drawn from studies with concerns regarding both the methodological quality and plausibility of the finding.

\section{Qualitative data analysis and synthesis}

For the review of barriers and facilitators, we will conduct the qualitative synthesis for types of facilitators and barriers related to uptake of testing. The aim of the synthesis is to enhance understanding of questions regarding "what works for whom and in what context" and to identify "barriers" and "facilitators" to the uptake of self-testing. The qualitative data analysis will base on thematic synthesis of qualitative research. Two authors will independently code key descriptive themes on types of facilitators and barriers, related with uptake of self-testing. We will discuss the resulting themes and sub-themes within the study team to examine their relationship to the review outcomes. The qualitative synthesis will then proceed by using the "descriptive themes" to develop "analytical themes," which will be interpreted in reference to the review aims.

\section{Discussion}

Achieving universal coverage of HIV testing for the general populations in Africa with scarce resources requires the implementation of innovative and cost-effective community-based HIV testing strategies, such as selftesting. The findings from this systematic review will inform on the knowledge gaps on the use of HIVST, yield and linkage to ARV treatment, incidence of social harms, and facilitators or barriers to uptake of HIVST among adults in Africa. We anticipate that our findings will guide the development of HIV self-testing policy, which is virtually non-existent at present in most African countries.

\section{Presenting and reporting of results}

This protocol will follow the Preferred Reporting Items for Systematic Review and Meta-Analysis Protocols (Additional file 4) 2015 Statement [42]. We will present the results of this review according to the Preferred Reporting Items for Systematic Reviews and MetaAnalyses (PRISMA) guidelines. A PRISMA flow chart will be produced to ensure transparency of the process [43]. A table of all included studies will be included in the final review, and the reasons for exclusion of studies will be clearly documented. Where statistical pooling is not possible, we will present the findings in narrative form using tables and figures to aid in data presentation.

\section{Interpretation of findings}

The results of the review will be discussed in the context of the quality of evidence, the limitations of the review, and the strengths of the findings, with emphasis on their implications for the current HIV self-testing practice and the potential for future research.

\section{Additional files}

Additional file 1: Appendix 1: Describing details of search strategy. (PDF $74 \mathrm{~kb}$ )

Additional file 2: Appendix 2: Systematic review data extraction form: Observational quantitative and qualitative studies. (PDF $159 \mathrm{~kb}$ )

Additional file 3: DATA EXTRACTION FORM. (DOCX $115 \mathrm{~kb}$ )

Additional file 4: PRISMA-P (Preferred Reporting Items for Systematic review and Meta-Analysis Protocols) 2015 checklist: recommended items to address in a systematic review protocol. (DOC 81 kb) 


\section{Abbreviations}

CASP: Critical Appraisal Skills Programme; CDSR: the Cochrane Database of Systematic Reviews; CENTRAL: the Cochrane Central Register of Controlled Trials; CerQual: certainty of qualitative evidence; EPOC: Effective Practice and Organisation of Care; GRADE: Grading of Recommendations Assessment, Development and Evaluation; GRADEpro: GRADE profiler; MeSH: Medical Subject Headings; PRISMA: Preferred Reporting Items for Systematic Reviews and Meta-Analyses; PRISMA-P: Preferred Reporting Items for Systematic Review and Meta-Analysis Protocols; RevMan: Review Manager.

\section{Competing interests}

The authors declare that they have no competing interests.

\section{Authors' contributions}

$\mathrm{BN}$ is the guarantor. All authors contributed to the conception and design of the protocol as follows. BN and CM conceived the study. BN and DD will conduct the search and data extraction under the supervision of LA. BN wrote the protocol under the supervision of $L A, A B$, and $C M$. All authors read the final draft of the manuscript and provided the feedback. All authors read and approved the final manuscript.

\section{Authors' information}

$\mathrm{BN}-\mathrm{MPH}$ is a PhD candidate in the School of Public Health and Family Medicine at the University of Cape Town, South Africa.

DD-BA is a MSC and PhD candidate in the School of Public Health and Family Medicine at the University of Cape Town, South Africa. $L A$ is a research fellow in the Vaccines for Africa Initiative, Institute of Infectious Disease and Molecular Medicine at the University of Cape Town, Cape Town, South Africa.

$A B$ is an associate professor of Public Health, Centre of Infectious Disease Epidemiology and Research University of Cape Town, South Africa. CM is the Head of Heath Systems Research Unit, Southern African Medical Research Council and Honorary Associate Professor of Public Health, in the School of Public Heath and Family Medicine, University of Cape Town, South Africa.

\section{Acknowledgements}

The authors acknowledge Ms. Dilshaad Brey the UCT Libraries, Health Sciences, and Information Services Librarian, who provide technical support and assisted in the planning of the search strategy. Dr. Mark Engel and Dr. Mahmoud Werfalli of Evidence-Based Medicine Research Support Unit, Faculty of Health Sciences at University of Cape Town; Darshini Govindasamy of South African Medical Research Council; and Claire Gudex of Department of Clinical Research, University of Southern Denmark, for their technical support.

\section{Author details}

'School of Public Heath and Family Medicine, University of Cape Town, Cape Town 7925, South Africa. ${ }^{2}$ Vaccines for Africa Initiative, Institute of Infectious Disease and Molecular Medicine, University of Cape Town, Cape Town 7925, South Africa. ${ }^{3}$ Department of Clinical Laboratory Sciences, Division of Medical Microbiology, Cape Town 7925, South Africa. ${ }^{4}$ Health Systems Research Unit, South African Medical Research Council, Cape Town, South Africa.

\section{Received: 9 November 2015 Accepted: 23 March 2016} Published online: 05 April 2016

\section{References}

1. UNAIDS. Global report:UNAIDS report on the global AIDS epidemic 2013. 2013. In.Geneva,Switzerland.

2. UNAIDS. UNAIDS gap report 2014, Geneva, Switzerland. 2014.

3. UNAIDS. How aids changed everything: MDG 6: 15 years, 15 lessons of hope from the aids response. Geneva: JOINT UNAIDS \& WHO; 2015.

4. UNAIDS. The GAP report. Geneva: 2014

5. The leDEA and ART cohort collaborationCochrane Collaboration. Immunodeficiency at the start of combination antiretroviral therapy in lowmiddle-, and high income countries. J Acquir Immune Defic Syndr. 2014 65(1):e8-e16. doi.10.1097/QAl.0b013e3182a39979.

6. Mukolo A, et al. Predictors of late presentation for HIV diagnosis: a literature review and suggested way forward. AIDS and Behavior 2013. 2013;17(1):5-30.

7. Johnson C, et al. Realizing the potential for HIV self-testing. AIDS Behav. 2014. doi:10.1007/s10461-014-0832-x.
8. Sharma M, et al. Systematic review and meta-analysis of community and facility-based HIV testing to address linkage to care gaps in sub-Saharan Africa. Nature. 2015;528:S77-S85.

9. Wong $\mathrm{V}$, et al. HIV self-testing in resource-limited settings: regulatory and policy considerations. AIDS Behavior. 2014;18 Suppl 4:S415-21.

10. Lee VJ, et al. User acceptability and feasibility of self-testing with HIV rapid tests. J ournal of Acquired Immune Deficiency Syndromes. 2007;45(4):449-53.

11. Njau B, et al. HIV testing preferences in Tanzania: a qualitative exploration of the importance of confidentiality, accessibility, and quality of service. BMC Public Health. 2014;14:838-47.

12. Xun $\mathrm{H}$, et al. Factors associated with willigness to accept oral fluid HIV rapid testing among most-at-risk populations in China. PLOS ONE. 2013;8(11):e80594.

13. Choko AT, et al. The uptake and accuracy of oral kits for HIV self-testing in high HIV prevalence setting: a cross-sectional feasibility study in Blantyre, Malawi. PLoS Med. 2011:8(10):e1001102.

14. Pai NP, et al. Supervised and unsupervised self-testing for HIV in high- and low-risk populations: a systematic review. PLoS Med. 2013;10(4):e1001414. doi:10.1371/journal.pmed.1001414.

15. Krause J, et al. Acceptability of HIV self-testing: a systematic literature review. BMC Public Health 2013. 2013;13:735.

16. Suthar $A B$, et al. Towards universal voluntary HIV testing and counselling: a systematic review and meta-analysis of community-based approaches. PLoS Med. 2013;10(8):e1001496. doi:10.1371/journal.pmed.1001496.

17. Mahler HR, et al. Voluntary medical male circumcision: matching demand and supply with quality and efficiency in a high-volume campaign in Iringa Region, Tanzania. PLoS Med. 2011;8(11):1-8.

18. Figueroa C, et al. Attitudes and acceptability on HIV self-testing among key populations: a literature review. AIDS Behavior. 2015;19:1949-65.

19. Walensky RP, Bassett IV. HIV self-testing and the missing linkage. PLoS Medicine. 2011:8(10):1-2.

20. Njau B, et al. A systematic review on uptake and yield of HIV self-testing among adults in Africa. 2015. Available from: http://www.crd.york.ac.uk/ PROSPERO_REBRANDING/display_record.asp?ID=CRD42015023935. Accessed 20 Nov 2015

21. Fylkenes K, Siziya S. A randomised trial on acceptability of voluntary counseling and testing. Trop Med Int Health. 2004;9(5):566-72.

22. Walensky RP, et al. The clinical impact and cost effectiveness of routine voluntary HIV screening in South Africa. J Acquir Immune Defic Syndr. 2011; 56(1):26.

23. Walensky RP, et al. Identifying undiagnosed human immunodeficiency virus: the yield of routine, voluntary inpatient testing. Arch Intern Med. 2002; 162(8):887-92

24. Govindasamy D, et al. Linkage to HIV, TB and non-communicable disease care from a mobile testing unit in Cape Town, South Africa. PLoS One. 2013;8(11):e80017.

25. Choko AT, et al. Uptake, accuracy, safety, and linkage into care over two years of promoting annual self-testing for HIV in Blantyre, Malawi: a community-based prospective study. PloS Med. 2015;12(9):e1001873.

26. WHO. Health expectancy is more important than life expectancy-message from the WHO director general on the World Health Report 1998. Geneva: WHO; 1998.

27. Eisinga A, Siegfried N, Clarke M. The sensitivity and precision of search terms in phases I, II, and III of the Cochrane highly sensitive search strategy for identifying reports of randomized trials in Medline in a specific area of health care-HIV/AIDS prevention and treatment interventions. Health Information and Library Journal. 2007;24:103-9.

28. Piennar $\mathrm{E}$, Grobler L, Busgeeth $\mathrm{K}$, et al. Developing a geographic search filter to identify randomised controlled trials in Africa: finding the optimal balance between sensitivity and precision. Health Info Libr J. 2011;28:210-15.

29. Noyes J, et al. Chapter 20: qualitative research and Cochrane reviews. In: Higgins JPT, Green S, editors. Cochrane Handbook of Systematic Reviews for Interventions Version 5.0.2[updated 2009]. 2009.

30. Flemming $\mathrm{K}$, Briggs M. Electronic searching to locate qualitaive reserach: evalaution of three strategies. J Adv Nurs. 2007:57(1):95-100.

31. Group CE. Concrane Effective Practice and Organization of Care(EPOC) Group. EPOC 'Risk of Bias' guideline. 2011. Available from: http://www.epoc. cochrane.org/en/handsearchers.html. Accessed 3 Mar 2016

32. Wells G, et al. The New Castle-Ottawa Scale(NOS) for assessing the quality of nonrandomosed studies in meta-analyses. 2011. Available from: http://www.ohri.ca/programs/clinical epidemiology/oxford.asp. Accessed 3 Mar 2016 
33. Cochrane Effective Practice and Organisation of Care Group. Risk of bias for studies with a separete control group (RCTs, CCTs, CBAs). 2009. Available from: http://epoc.cochrane.org/epoc-resources-review-authors2009. Accessed 3 Mar 2016.

34. Higgins JPT, Green SES. Cochrane handbook for systematic reviews of interventions 4.2.5 (updated May 2005). 2005. Vol. In the Cochrane Library, issue 3 .

35. NICE. Public health guidance manual. 2013. Available from: http:// publications.nice.org.uk/methods-for-the-devalopment-of-nice-public-healthguidance-thord-edition-pmg4/appendix-H-quality-appraisal-checklistqualitative-studies. Accessed 3 Mar 2016.

36. Critical Appraisal Skills Programme(CASP). 10 questions to help you make sense of qualitative research. England: Critical Appraisals Skills Programme 2010; 2010. Available from: http://www.casp-uk.net/wp-content/uploads/ 2011/11/CASP Qualitative Appraisal Checklist 140ct10.pdf. Accessed on 3rd Marc,2016.

37. RevMan. The Nordic Cochrane centre, The Cochrane Collaboration. Coppenhagen: The Nordic Cochrane centre,The Conchrane Collaboration,2003; 2003. Review Manager (REVMan). 4.2 for Windows.

38. Campbell M, Grimshaw J, Steen N. Sample size calculations for cluster randomised trials. Changing Proffesional Practice in Europe Group(EU BIOMED II Concerted Action). J. Health Services Research and Policy. 2000;5:12-6.

39. Donner A, Piaggio G, Villar J. Statistical methods for the meta-analysis of cluster randimization trials. Statistical Methods in Medical Research. 2001;10:325-38.

40. Guyatt GH, et al. GRADE: an emerging consesus on rating quality of evidence and strength of recommendations. BMJ. 2008;336:924-6.

41. Balshem $\mathrm{H}$, et al. GRADE guidelines: 3. Rating the quality of evidence. J. Clinical Epidemiology. 2011;64(4):401-6.

42. Shamseer $L$, et al. Preferred reporting items for systematic review and meta-analysis protocals(PRISMA-P)2015:elaboration and explanation. BMJ. 2015;349:97647.

43. Liberati A, et al. The PRISMA statement for reporting systematic reviews and meta-analyses of studies that evaluate health care interventions: explanation and elaboration. PLoS Med. 2009;6(7):e1000100.

\section{Submit your next manuscript to BioMed Central and we will help you at every step:}

- We accept pre-submission inquiries

- Our selector tool helps you to find the most relevant journal

- We provide round the clock customer support

- Convenient online submission

- Thorough peer review

- Inclusion in PubMed and all major indexing services

- Maximum visibility for your research

Submit your manuscript at www biomedcentral.com/submit

) Biomed Central 